\title{
Muscular Damage and Kidney Function in Rugby Players after Daily Whole Body Cryostimulation
}

\author{
Giovanni Lombardi, ${ }^{1}$ Alessandra Colombini, ${ }^{1}$ Simone Porcelli, ${ }^{2}$ Clara Mauri, ${ }^{3}$ \\ Viviana Zani, ${ }^{4}$ Felice Bonomi, ${ }^{4,5}$ Gianluca Melegati, ${ }^{3}$ and Giuseppe Banfi ${ }^{1,6}$ \\ ${ }^{1}$ Laboratory of Experimental Biochemistry and Molecular Biology, I.R.C.C.S. Istituto Ortopedico Galeazzi, Via R. Galeazzi, \\ 4-20161 Milano, Italy \\ ${ }^{2}$ Institute of Bioimaging and Molecular Physiology, National Research Council, Via Fratelli Cervi, \\ 93-20090 Segrate, Italy \\ ${ }^{3}$ Department of Multifunctional Rehabilitation, I.R.C.C.S. Istituto Ortopedico Galeazzi, Via R. Galeazzi, 4-20161 Milano, Italy \\ ${ }^{4}$ Centre of Systemic Cryotherapy, Poliambulatorio Bongi, Via Magli, 25034 Orzinuovi, Italy \\ ${ }^{5}$ Cardiovascular Department, O.U Cardiology, Humanitas Gavazzeni, Via Mauro Gavazzeni, 21-24125 Bergamo, Italy \\ ${ }^{6}$ Department of Biomedical Sciences for Health, University of Milano, Via R. Galeazzi, 4-20161 Milano, Italy
}

Correspondence should be addressed to Giovanni Lombardi; giovanni.lombardi@grupposandonato.it

Received 13 February 2014; Accepted 27 May 2014; Published 12 June 2014

Academic Editor: Beat Knechtle

Copyright ( 2014 Giovanni Lombardi et al. This is an open access article distributed under the Creative Commons Attribution License, which permits unrestricted use, distribution, and reproduction in any medium, provided the original work is properly cited.

\begin{abstract}
Muscular damage, consequent to strenuous activities, could exceed the recovery potential of muscles and determine renal failure. Whole body cryostimulation is a cold-based therapy used to improve recovery or overcome fatigue symptoms. This study aimed to evaluate the effects of repeated sessions of cryostimulation on muscle damage, renal function, and their relationship. Serum samples, from 27 elite rugby players, under training, before and after 2 sessions/day of cryotherapy over 7 days, were tested for markers of muscular (creatine kinase, lactate dehydrogenase, and aspartate aminotransferase) and renal (creatinine, cystatin C) functions. eGFR was calculated with two formulas based on either serum creatinine concentration (MDRD) or serum creatinine and cystatin C concentrations (creatinine/cystatin C CKD EPI). Pre- and posttreatment differences were determined by Wilcoxon's test; correlations were evaluated with Spearman's test. Cryostimulation helped muscular recovery (increased lactate dehydrogenase activity $(P<0.001)$ and the stabile creatine kinase and aspartate aminotransferase activities). Creatinine was unaffected while cystatin $\mathrm{C}$ was increased $(P=0.013)$. Creatinine-based eGFR was not affected by cryostimulation, while creatinine/cystatin Cbased eGFR showed a slight decrease $(P=0.015)$. eGFR and muscular biomarkers were not correlated, suggesting a real recovery effect of cryotherapy. Cystatin C seemed more sensible than creatinine in evaluating the kidney function.
\end{abstract}

\section{Introduction}

Exercise rises muscle metabolic parameters (creatine kinase (CK), lactate dehydrogenase (LDH), and aspartate aminotransferase (AST)) and, thus, their interpretation could be helpful in monitoring recovery from normal training, overtraining, or, even, from muscular trauma [1]. If the exercise intensity is strenuous, the muscle can be damaged and this is evidenced by increasing soreness, weakness, and tenderness along with strength loss. The muscular damage is molecularly marked by increased blood levels of $\mathrm{CK}, \mathrm{LDH}$, and myoglobin
[2]. Myoproteins are released from muscle cells due to the increased leakage from membrane as a consequence of the contractile activity. One of the main causes of increased membrane permeability or susceptibility to breaking is the action of exercise-generated free radicals [3].

Serum activity of CK is one of the most studied markers for monitoring muscular status and recovery in sports medicine. Its increase depends on sport discipline and performance length. Moreover, athletes show higher, even doubled, basal values of CK than their sedentary counterparts, as reported, at rest, after $24-48 \mathrm{~h}$ from exercise $[4,5]$. In athletes, 
CK activity should be measured on a short-term basis to monitor the recovery during training: an efficient recovery is, in fact, marked by a postexercise return to basal of CK, while persistent high values can be a sign of trauma, overtraining, or muscular pathology, as demonstrated in overtrained rugby players [6]. However, muscle recovery cannot be assessed through the evaluation of the changes in serum CK levels only because no correlation has been found between serum enzyme leakage and muscular performance impairment after exercise: overreaching should be suspected when there is a persistent increase in $\mathrm{CK}$, but a reduction in exercise tolerance should also be manifest [7].

For what concerns LDH activity, muscle biopsies have evidenced different activity of total $\mathrm{LDH}$ and $\mathrm{LDH}$ isoenzymes in endurance and strength athletes, with the former having lower total LDH activity, with the prevalence of LDH12 isoenzymes, compared with the latter, who showed higher total LDH activity and, prevalently, of LDH5 [8].

Monitoring both LDH and CK activities provides useful information on the state of the muscle and on its biochemical adaptation to the physical load, since subjects with persistently elevated CK activity also have altered LDH profiles [8].

AST is another molecular marker of muscular status, often analyzed in association with $\mathrm{CK}$ and LDH. It is mainly produced by the liver but, also, in considerable amounts in other tissues, including the muscles, and it is released from activated muscles during physical exercise [9]. The potential effectiveness of various recovery methodologies on serum CK activity was studied by Gill and coworkers. In that study, the active postcompetition recovery, in rugby players, was obtained with one of the following methods: passive recovery, stationary bicycle, compressive garments, and water contrast (repetitions of lower limb immersions for 1 minute in cold water at $8-10^{\circ} \mathrm{C}$ and 2 minutes in hot water at $40-42^{\circ} \mathrm{C}$, for a total of 9 minutes). CK activities were shown to be downed efficiently only by passive recovery [10]. Contrariwise, in rugby players belonging to the Italian rugby national team, the combination of cycling and legs water immersion effectively stabilize the posttraining CK levels [11].

Whole body cryotherapy (WBC) is a peculiar form of cold-based therapy entered about 30 years ago in the clinical practice for the symptomatic treatment in rheumatoid arthritis. It consists of a short (2-3 minutes) whole-body exposure to extremely cold air $\left(-110^{\circ}\right.$ to $\left.-140^{\circ} \mathrm{C}\right)$. Currently, this therapeutic approach is widely used in sports medicine to accelerate muscular recovery after injuries or to overcome fatigue symptoms $[12,13]$. Due to the demonstrated benefits in athletes, WBC (in this case better defined as whole body cryostimulation) acquired, in the last years, a growing popularity among athletes and sports physicians. Indeed, though not so numerous, there are evidences about positive effects of WBC in enhancing the cardiovascular function, amelioration of muscular activation and recovery, limitation of sport-induced haemolysis, and improvement of the proand anti-inflammatory balance and oxidative status [12].

Only a single work evaluates the effect of WBC on CK activity; however, it was based on a single session of $\mathrm{WBC}$ as a tool for recovery from exercise-induced muscular damage [14]. To our knowledge there are no published papers
TABLE 1: Anthropometrical features of the study cohort.

\begin{tabular}{lccc}
\hline Data & Pre-WBC & Post-WBC & $P$-value \\
\hline$N$ & 27 & 27 & \\
Height $(\mathrm{m})$ & $1.85(1.78-1.97)$ & $/$ & $/$ \\
Weight $(\mathrm{kg})$ & $102.50(85.39-116.78)$ & $101.80(85.54-114.58)$ & 0.394 \\
BMI $\left(\mathrm{kg} / \mathrm{m}^{2}\right)$ & $29.07(25.79-33.11)$ & $28.86(25.80-33.17)$ & 0.354 \\
\hline
\end{tabular}

The table summarizes the main anthropometrical features of the rugby players recruited in the study $(n=27)$ before and after the treatment. Data are shown as median (5th-95th percentile).

analyzing the effects of a cycle of WBC sessions on the markers of muscular damage and the renal function. Indeed, a relationship has been demonstrated between CK activity in serum and renal function. When the exercise-induced damage exceeds the recovery potential of the muscle, as often observed in ultraendurance performances, a clinical condition named exertional rhabdomyolysis could emerge that can, in turn, determine a renal failure [15]. While CK is cleared from circulation by the reticuloendothelial system, myoglobin is cleared by the kidney: in the case of massive muscular damage results high blood myoglobin levels and myoglobinuria; myoglobin can precipitate in the kidney tubules potentially resulting in acute renal failure especially in environmental conditions of heat stress and dehydration [16].

Once demonstrating this relationship, in this study we evaluated the effects of WBC on the behavior of the markers of muscular damage and the renal function, in professional rugby players.

\section{Materials and Methods}

2.1. Subjects. The study population was composed of 27 males professional rugby players, belonging to the Italian National Rugby Team participating in the 2012 summer training camp (May 21st to 27th), at the end of the competitive season. The median age was 24.8 ranging from 21.4 to 31.9. Anthropometrical features of the study cohort (age, height, weight, and body mass index (BMI)) were recorded (Table 1).

The cryostimulation (WBC) entered in the team practice as a part of the end season-recovery, while the blood samplings were part of the routine medical checks. The data reported in the present paper are, thus, the results of an observational cohort protocol. The only exclusion criterion, for this highly selected homogeneous population, was any acute condition preventing the participation to the entire camp or to any activity of the camp.

The study was approved by the IRB (ASL Città di Milano) in accordance with the Helsinki declaration. All the subjects were informed about risks and benefits of the treatment protocol and they all gave their consent to the use of data for scientific purposes.

2.2. Methods. The WBC treatment protocol was performed at the Centre of Systemic Cryotherapy (Poliambulatorio Bongi 
S.r.l., Orzinuovi, Italy) consisting of 2 sessions per day (the first in the morning, after the morning training session, and the second in the evening, after the afternoon training session) for 7 consecutive days. Every single session consisted of 30 -second-long preconditioning at $-60^{\circ} \mathrm{C}$ and a 3 -min long exposure at $-140^{\circ} \mathrm{C}$. During the treatment, the subjects were minimal clothed and, to avoid frostbite, they wore shorts (bathing suit), socks, clogs or shoes, surgical mask, gloves, and hat (or headband) covering the auricles. Any sweat was dried before entering the cryochamber, where the air was clear and dry. While into the cryochambers subjects were asked to walk within the chamber, to maintain the finger in motion and to avoid breath holding. The system was automatically controlled; safety personnel and medical doctors were always present. The athletes were never submitted to WBC; only 3 of them underwent WBC about five years earlier.

The training week consisted of two sessions, two hours each, per day. In the first three days, the morning session consisted of (i) warm-up, (ii) weight training, and (iii) plyometrics; the afternoon session consisted of (i) warm-up, (ii) rugby skills, and (iii) interval training. On the fourth day, athletes were submitted to two light and easy sessions of interval training, while on the fifth day they were off. On the sixth day athletes underwent two sessions of free sport activities while, on the last day, there were scheduled two sessions of slow and long distance exercises.

Diet regimen was strictly defined by team physicians and diet included a correct quantity of iron and folates.

Two blood drawings were performed, the former prior to the start of the training camp and WBC protocol, while the latter was performed the day after the end of the camp. Blood samples were collected in the morning (08:00 a.m.), under standard conditions, on fasting subjects, at rest in sitting position, by antecubital venipuncture, into $7 \mathrm{~mL}$ plain tubes (BD SSTII Advance; Becton-Dickinson, Franklin Lakes, NJ, USA) for clinical chemistry testing. Creatinine concentration was analyzed by the enzymatic method; CK, LDH, and AST activities were measured on a Siemens Advia 1800 (Siemens, Tarrytown, NY, USA); and the cystatin C test was performed by nephelometry on a Siemens BN ProSpec (Siemens). During the study period, the analyzers were regularly calibrated and continuously controlled by internal and external quality control schemes. The imprecision of the methods were $0.6 \%$ to $1.1 \%$ for creatinine, $2.7 \%$ to $3.0 \%$ for $\mathrm{CK}, 1.2 \%$ to $1.7 \%$ for $\mathrm{LDH}$, for AST $2.3 \%$ to $3.3 \%$, and for cystatin C $2.4 \%$ to $4.3 \%$.

2.3. Estimation of the Glomerular Filtration Rate. Estimated GFR (eGFR) based on creatinine concentrations was calculated by the means of both creatinine- and creatinine and cystatin $\mathrm{C}$-based equations. The reported equations are reported with the correcting factors (ethnicity, gender, creatinine [Scr], and cystatin C [Scys] concentration) already added/eliminated, according to the instructions provided by the authors.

(i) Modification of Diet in Renal Disease (MDRD), recommended by the Kidney Disease Outcomes Quality Initiative (K/DOQI) guidelines [17, 18]:

$$
\begin{aligned}
\mathrm{mL} / \min / 1.73 \mathrm{~m}^{2} \\
=186 \times\left[\mathrm{Scr}\left(\frac{\mu \mathrm{mol}}{\mathrm{L}}\right) \times 0.011312\right]^{-1.154} \times[\text { age }]^{-0.203} ;
\end{aligned}
$$

(ii) CKD-EPI creatinine-cystatin $\mathrm{C}([\mathrm{Scr}]>0.9 \mathrm{mg} / \mathrm{dL})$ [19]:

$$
\begin{aligned}
& \text { if }[\text { Cystatin C }] \leq 0.8: \mathrm{mL} / \mathrm{min} / 1.73 \mathrm{~m}^{2} \\
& =135 \times\left(\frac{\mathrm{Scr}}{0.9}\right)^{-0.601} \times\left(\frac{\mathrm{Scys}}{0.8}\right)^{-0.375} \\
& \quad \times 0.995^{\text {Age }}, \\
& \text { if }[\text { Cystatin C }]>0.8: \mathrm{mL} / \mathrm{min} / 1.73 \mathrm{~m}^{2} \\
& =135 \times\left(\frac{\text { Scr }}{0.9}\right)^{-0.601} \times\left(\frac{\text { Scys }}{0.8}\right)^{-0.711} \\
& \quad \times 0.995^{\text {Age }} .
\end{aligned}
$$

2.4. Statistical Analysis. Statistical analysis was performed by GraphPad Prism v5.0 software (GraphPad Software Inc., La Jolla, CA, USA). Values, in the descriptive analysis, are expressed as the median (5th-95th percentile). The comparisons of before treatment versus after treatment were performed by Wilcoxon's matched pairs test. The comparison between the eGFR outputs obtained by the two formulas was performed at each time-point by the means of two-tailed Wilcoxon's signed rank paired test. Correlation analysis, by the means of two-tailed Spearman's correlation test, was applied to evaluate the relationships existing among the trend (in terms of difference between post-WBC and pre-WBC) of the biomarkers and those of the renal function indexes. The significance level was set at 0.05 .

\section{Results}

3.1. Anthropometrical Features. The anthropometrical features of the study cohort are reported in Table 1 . As it is possible to note, the whole-body cryostimulation has no significant effects on the parameters here analyzed. Particularly, no appreciable modifications in weight and BMI were found.

3.2. Biomarkers Serum Levels. A significant increase was found in the trends of LDH activity which rose from 172.0 (136.2-214.0) U/L before WBC to 190.0 (152.2-236.0) U/L after the cryostimulation $(P<0.001)$. Contrarily, CK and AST activities did not change significantly and the recorded values were 178.00 (88.40-910.60) U/L before WBC and $152.00(84.20-739.40) \mathrm{U} / \mathrm{L}$ after the treatment for $\mathrm{CK}$ $(P=0.127)$ and $25.00(16.40-44.60) \mathrm{U} / \mathrm{L}$ to $28.00(17.00-$ $51.60) \mathrm{U} / \mathrm{L}$ for AST $(P=0.075)$. Pre- and post-WBC LDH, $\mathrm{CK}$, and AST activities are reported in Figure 1.

For what concerns the marker of renal function, different trends were found between creatinine and cystatin C. Indeed, serum creatinine was not substantially modified 

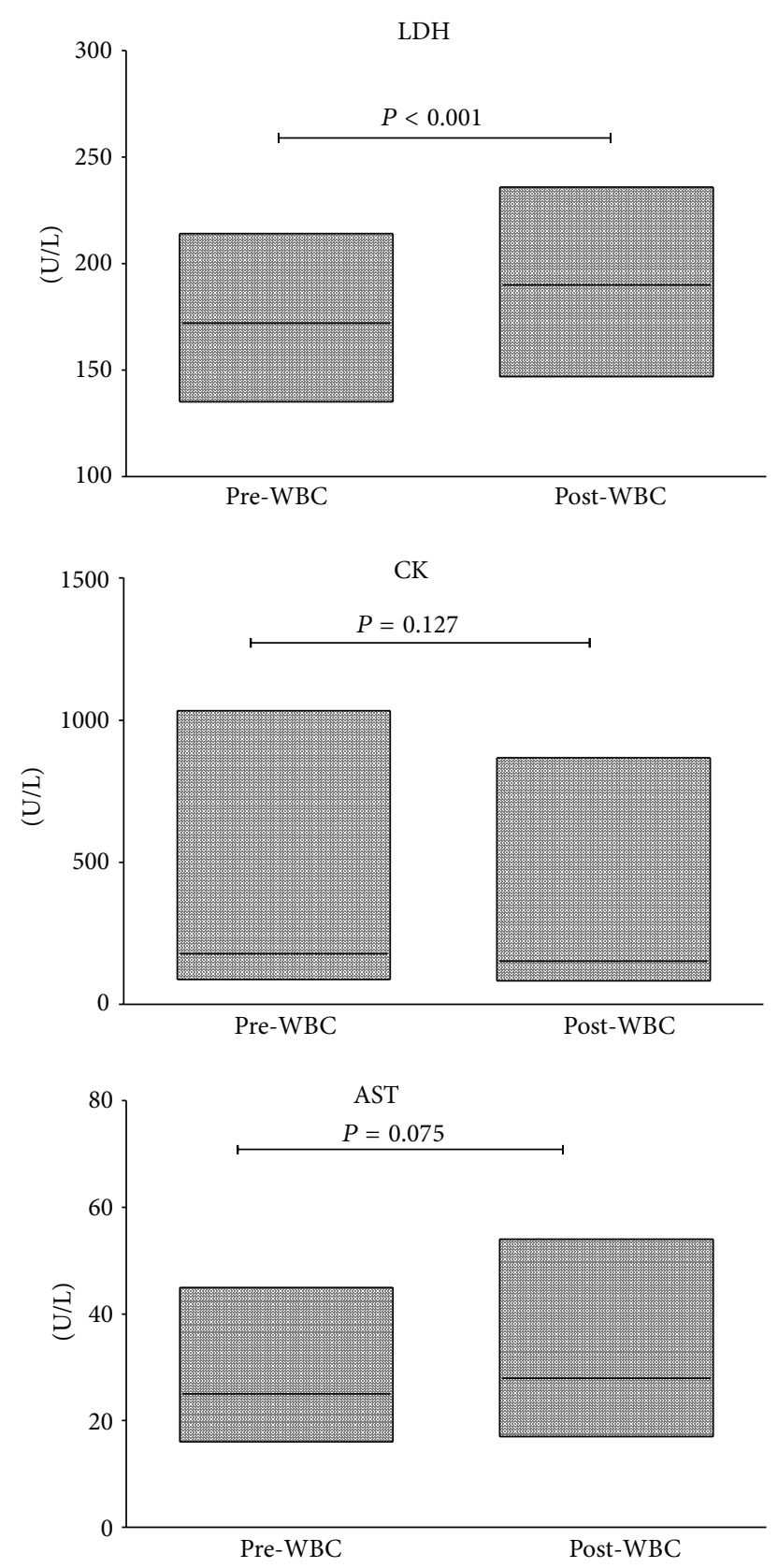

FIGURE 1: Changes in the levels of muscular damage biomarkers following WBC. The figure shows the changes in the serum activities of CK, LDH, and AST pre- and post-WBC. The box and whisker plot show the median and the range for each parameter at the two timepoints. The $P$ value for the paired $t$-test is reported.

by the treatment $(1.02(0.90-1.23) \mathrm{mg} / \mathrm{dL}$ pre-WBC and 1.03 $(0.89-1.27) \mathrm{mg} / \mathrm{dL}$ post-WBC, $P=0.850)$, while cystatin $\mathrm{C}$ was found slightly but significantly increased $(0.73(0.64-$ $0.84) \mathrm{mg} / \mathrm{L}$ pre-WBC and $0.75(0.66-0.90) \mathrm{mg} / \mathrm{L}$ post-WBC; $P=0.013)$.

Creatinine and cystatin $\mathrm{C}$ values are reported in Figure 2.

3.3. Estimated Glomerular Filtration Rate. The two formulas used for calculating the eGFR gives the following results:
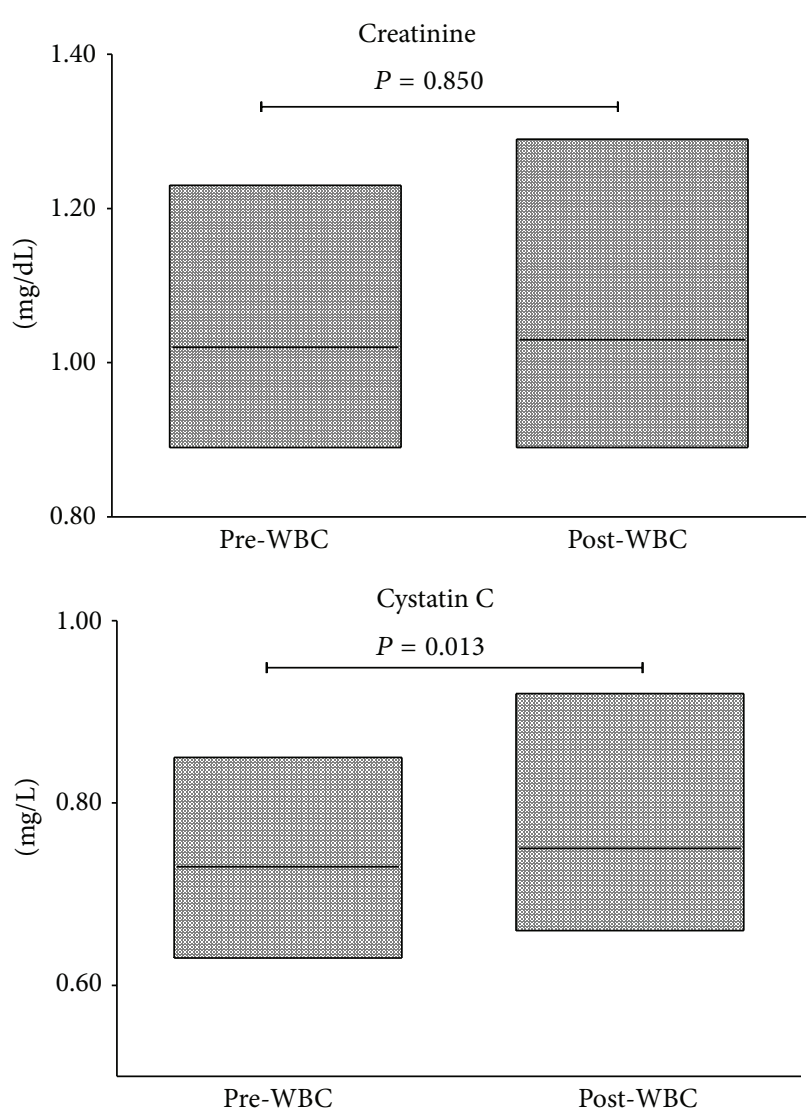

FIGURE 2: Changes in the levels of renal function biomarkers following WBC. The figure shows the changes in the serum concentrations of creatinine and cystatin C pre- and post-WBC. The box and whisker plot shows the median and the range for each parameter at the two time-points. The $P$ value for the paired $t$-test is reported.

90.35 (76.57-108.10) $\mathrm{mL} / \mathrm{min}$ pre-WBC and 88.14 (68.03$117.60) \mathrm{mL} / \mathrm{min}$ post $\mathrm{WBC}$ for the MDRD equation and $113.00(97.40-126.50) \mathrm{mL} / \mathrm{min}$ pre-WBC and $106.46(88.40-$ 131.10) $\mathrm{mL} / \mathrm{min}$ post-WBC for the creatinine/cystatin C CKD-EPI formula. No differences were found in the MDRD before and after the WBC $(P=0.207)$ while the CKD-EPI creatinine and cystatin $C(P=0.015)$ formula indicated a slight but significant decrease in eGFR between pre- and postWBC.

At both time-points the mathematical outputs of the two formulas were significantly different $(P<0.001)$.

3.4. Correlations Analysis. There were found correlation among the muscular markers between LDH and CK $(r=$ $0.43, P=0.025)$ and CK and AST $(r=0.74, P<0.001)$. Creat was correlated with MDRD $(r=-0.85, P<0.001)$ and CKDEPI Scr Scys $(r=-0.77, P<0.001)$. No other correlation was found for the other comparisons. Particularly, no correlations were found for CK with Creat $(r=0.27$, $P=0.173)$, cyst C $(r=0.10, P=0.613)$, MDRD $(r=-0.31$, $P=0.117)$, and CKDEPI Scr Scys $(r=-0.36, P=0.065)$. 


\section{Discussion}

The behavior of laboratory parameters in athletes, especially in professional athletes, has not been extensively studied and described [1]. Among the laboratory parameters, LDH, CK, and AST are surely the most studied ones in athletes, since their strict link with muscle health and recovery. However, despite the increasingly widespread use of WBC, especially in sports medicine, little is known about the physiological response of healthy subjects to this practice [12].

Some previous reports advocated a positive effects of $\mathrm{WBC}$ on muscular recovery after normal training or overload: a reduction of the proinflammatory markers, associated with an increase in the anti-inflammatory potential [20], an enhancement of muscle fibers repair (marked by decreases in $\mathrm{LDH}$ and $\mathrm{CK}$ ) $[5,6]$, and a reduction of microinjuries to muscle fibers caused by exercise (shown by a decrease in CK serum concentration) [21].

Concerning the muscular damage markers, our present results demonstrated that, after the WBC treatment, only LDH was significantly increased, while CK and AST remained unchanged. Since it was previously reported, in professional rugby players, that a short-term training induced significant increases in both $\mathrm{LDH}$ and CK $[9,11]$, the present data confirm the effectiveness of WBC in attenuating the muscular damage and stimulating recovery.

Recent works, by our groups, investigated the effects of different kind of physical activity on this interplay demonstrating that the modification of renal function after prolonged physical activity, described by creatinine, cystatin C, or eGFR variations, are generally transient and they will recover promptly because of the progressive adaptation of the kidneys and the physiological renal balance of the effects such physical activity has on the metabolism [22,23]. Nevertheless, in 29 trained women, performing a strenuous resistance exercise session, a certain degree of renal impairment is observed, testified by serum creatinine increase, urinary creatinine and eGRF decrease, and a significant negative correlation between CK activity and eGFR after exercise [16]. To our knowledge, however, no previous data have been published regarding the effects of WBC on renal function, nor evaluating the effects of the cryostimulation on the relationship between the markers of muscular damage and the renal function.

In the present work we found that WBC only minimally affects the eGFR and no correlations were found between the $\triangle \mathrm{eGFR}$ and the trends of muscular damage biomarkers. To test the possible variations in eGFR, we used two different equations: the MDRD recommended by the Kidney Disease Outcomes Quality Initiative (K/DOQI) guidelines [17, 18] and creatinine and cystatin C-based modification of the CKD EPI [19]. As known, the results of different formula may be even appreciably different [19]. Indeed, in the present work we confirm these differences.

Contrarily to the MDRD, the creatinine/cystatin Cbased CKD-EPI equation indicated a significant, even if slight, decrease after the cryostimulation (about 4\%). This poor agreement between the eGFR formulas confirmed the hypothesis of their poor utility in the kidney function assessment in athletes $[23,24]$. A significant increase was found in cystatin C levels, while no modification in serum creatinine was recorded. Noteworthy our pre- and post-WBC cystatin C concentrations were comparable, or even slightly lower, to those reported in a previous investigation performed on the same kind of subjects [25]. Notably, none of the athletes exceed the upper limit established for cystatin C, while 2 out of 27 subjects, after WBC, exceed the upper limit of $110 \mu \mathrm{mol} / \mathrm{L}$ established for creatinine. The use of equations to assess kidney function has evident practical advantages [26]. However, they have been mainly validated in patients suffering from late-stage chronic kidney disease (CKD), while no validation has been attempted in healthy individuals. As a general evidence, serum creatinine-based eGFR equations showed a larger uncertainty for high than for low GFR, mainly due to bias and measurement imprecision in the determination of the creatinine level [26]. Therefore, the general recommendation is to report only eGFR = $60 \mathrm{~mL} / \mathrm{min} / 1.73 \mathrm{~m}^{2}$ for eGFR above this level [26]. This limit has been demonstrated to be too low for some categories of athletes such as rugby players [25]. Another important limit to the use of creatinine-based equations, to calculate the GFR in athletes, is the need for correction for BSA to avoid eGFR overestimation in subjects having high mass, such as rugby players, and the contrary in subjects having low mass [27]. The use of cystatin $\mathrm{C}$ overcomes this matter since it is not dependent on muscular mass [25]. However, due to the lack of any reference material as calibrators for assays, cystatin C-based equations have only been proposed [28]. At this regard, our previous works have demonstrated that cystatin $\mathrm{C}$ was a reliable parameter for monitoring the healthy status of the athletes $[23,25]$. Although previous studies demonstrated that the effects of WBC become evident after multiple consecutive sessions, we hypothesize that there is an additive effect due to continuous exposure. Indeed, we have recently demonstrated, in the same population of rugby players, that the salivary steroid hormone profile is modified already after 2 sessions of WBC, performed on the same day. The same modifications are present, although amplified, after 14 WBC sessions over a week [29].

The main limitation of this study is the lack of a control group; this lack does not allow for discriminating the real effects of WBC from the cumulative effects of training and WBC. However, as stated above, the results of previous studies, performed on equivalent populations, provide a possible term of comparison that could partially overcome this lack. Unfortunately, this limitation is inevitable since elite athletes train together at the camps and undergo the same interventions. However, previous similar studies [30] were drawn up in the same way. Nonetheless, it is vital to confirm the present findings with case-control studies.

\section{Conclusions}

The main findings of this study can be summarized as follows: (i) WBC attenuated the effects of training in fact of $\mathrm{CK}$ activity levels were unchanged after the training camp; (ii) in general, WBC treatment did not induce appreciable variations in the eGFR, and, thus, its effects on muscular 
recovery could be considered a matter of fact; (iii) serum creatinine was not affected by WBC treatment; (iv) cystatin C-based formulas seemed to be more sensible in detecting even small changes in eGFR.

\section{Conflict of Interests}

The authors declare that there is no conflict of interests regarding the publication of this paper.

\section{Acknowledgments}

This work has been partially funded by the Italian Ministry of Health. The authors are indebted to the whole Italian Rugby National Team for the availability and the logistics. Gianluca Melegati belongs to Medical Board, Federazione Italiana Rugby-F.I.R., Roma, Italy.

\section{References}

[1] G. Banfi, A. Colombini, G. Lombardi, and A. Lubkowska, "Metabolic markers in sports medicine," Advances in Clinical Chemistry, vol. 56, pp. 1-54, 2012.

[2] J. D. Warren, P. C. Blumbergs, and P. D. Thompson, "Rhabdomyolysis: a review," Muscle and Nerve, vol. 25, no. 3, pp. 332347, 2002.

[3] P. Brancaccio, N. Maffulli, and F. M. Limongelli, "Creatine kinase monitoring in sport medicine," British Medical Bulletin, vol. 81-82, no. 1, pp. 209-230, 2007.

[4] H. K. Vincent and K. R. Vincent, "The effect of training status on the serum creatine kinase response, soreness and muscle function following resistance exercise," International Journal of Sports Medicine, vol. 18, no. 6, pp. 431-437, 1997.

[5] G. Lippi, G. Brocco, G. L. Salvagno, M. Montagnana, F. Dima, and G. C. Guidi, "High-workload endurance training may increase serum ischemia-modified albumin concentrations," Clinical Chemistry and Laboratory Medicine, vol. 43, no. 7, pp. 741-744, 2005.

[6] A. J. Coutts, P. Reaburn, T. J. Piva, and G. J. Rowsell, "Monitoring for overreaching in rugby league players," European Journal of Applied Physiology, vol. 99, no. 3, pp. 313-324, 2007.

[7] I. Margaritis, F. Tessier, F. Verdera, S. Bermon, and P. Marconnet, "Muscle enzyme release does not predict muscle function impairment after triathlon," Journal of Sports Medicine and Physical Fitness, vol. 39, no. 2, pp. 133-139, 1999.

[8] P. Brancaccio, F. M. Limongelli, and N. Maffulli, "Monitoring of serum enzymes in sport," British Journal of Sports Medicine, vol. 40, no. 2, pp. 96-97, 2006.

[9] G. Banfi and P. Morelli, "Relation between body mass index and serum aminotransferases concentrations in professional athletes," Journal of Sports Medicine and Physical Fitness, vol. 48, no. 2, pp. 197-200, 2008.

[10] N. D. Gill, C. M. Beaven, and C. Cook, "Effectiveness of postmatch recovery strategies in rugby players," British Journal of Sports Medicine, vol. 40, no. 3, pp. 260-263, 2006.

[11] G. Banfi, G. Melegati, and P. Valentini, "Effects of cold-water immersion of legs after training session on serum creatine kinase concentrations in rugby players," British Journal of Sports Medicine, vol. 41, no. 5, article 339, 2007.
[12] G. Banfi, G. Lombardi, A. Colombini, and G. Melegati, "Wholebody cryotherapy in athletes," Sports Medicine, vol. 40, no. 6, pp. 509-517, 2010.

[13] L. Bettoni, F. G. Bonomi, V. Zani et al., "Effects of 15 consecutive cryotherapy sessions on the clinical output of fibromyalgic patients," Clinical Rheumatology, vol. 32, no. 9, pp. 1337-1345, 2013.

[14] C. Hausswirth, J. Louis, F. Bieuzen et al., "Effects of wholebody cryotherapy vs. far-infrared vs. passive modalities on recovery from exercise-induced muscle damage in highlytrained runners," PLoS ONE, vol. 6, no. 12, Article ID e27749, 2011.

[15] K. P. Skenderi, S. A. Kavouras, C. A. Anastasiou, N. Yiannakouris, and A.-L. Matalas, "Exertional rhabdomyolysis during a 246-km continuous running race," Medicine and Science in Sports and Exercise, vol. 38, no. 6, pp. 1054-1057, 2006.

[16] M. Machado, E. N. Zini, S. D. Valadão, M. Z. Amorim, T. Z. Barroso, and W. de Oliveira, "Relationship of glomerular filtration rate and serum CK activity after resistance exercise in women," International Urology and Nephrology, vol. 44, no. 2, pp. 515-521, 2012.

[17] National Kidney Foundation, "K/DOQI clinical practice guidelines for chronic kidney disease: evaluation, classification, and stratification," American Journal of Kidney Diseases, vol. 39, no. 39, pp. S1-S266, 2002.

[18] E. J. Lamb, C. R. V. Tomson, and P. J. Roderick, "Estimating kidney function in adults using formulae," Annals of Clinical Biochemistry, vol. 42, no. 5, pp. 321-345, 2005.

[19] L. A. Inker, C. H. Schmid, H. Tighiouart et al., "Estimating glomerular filtration rate from serum creatinine and cystatin C," The New England Journal of Medicine, vol. 367, no. 1, pp. 20-29, 2012.

[20] A. Lubkowska, Z. Szyguła, D. Chlubek, and G. Banfi, "The effect of prolonged whole-body cryostimulation treatment with different amounts of sessions on chosen pro-and antiinflammatory cytokines levels in healthy men," Scandinavian Journal of Clinical and Laboratory Investigation, vol. 71, no. 5, pp. 419-425, 2011.

[21] A. Wozniak, B. Wozniak, G. Drewa, C. Mila-Kierzenkowska, and A. Rakowski, "The effect of whole-body cryostimulation on lysosomal enzyme activity in kayakers during training," European Journal of Applied Physiology, vol. 100, no. 2, pp. 137142, 2007.

[22] A. Colombini, R. Corsetti, M. MacHado et al., "Serum creatine kinase activity and its relationship with renal function indices in professional cyclists during the Giro d'Italia 3-week stage race," Clinical Journal of Sport Medicine, vol. 22, no. 22, pp. 408-413, 2012.

[23] A. Colombini, R. Corsetti, R. Graziani, G. Lombardi, P. Lanteri, and G. Banfi, "Evaluation of creatinine, cystatin C and eGFR by different equations in professional cyclists during the Giro d'Italia 3-weeks stage race," Scandinavian Journal of Clinical and Laboratory Investigation, vol. 72, no. 2, pp. 114-120, 2012.

[24] G. Banfi, J. Sloand, M. Shelly, M. Del Fabbro, A. Barassi, and G. V. M. d'Eril, "Limitations of Cockcroft-Gault and MDRD formulas in estimating GFR among top-level rugby players," Journal of Nephrology, vol. 25, no. 6, pp. 1047-1053, 2012.

[25] G. Banfi, M. Del Fabbro, G. M. d'Eril, and G. Melegati, "Reliability of cystatin $\mathrm{C}$ in estimating renal function in rugby players," Annals of Clinical Biochemistry, vol. 46, no. 5, p. 428, 2009. 
[26] G. L. Myers, W. G. Miller, J. Coresh et al., "Recommendations for improving serum creatinine measurement: a report from the Laboratory Working Group of the National Kidney Disease Education Program," Clinical Chemistry, vol. 52, no. 1, pp. 5-18, 2006.

[27] R. Milic, A. Colombini, G. Lombardi, P. Lanteri, and G. Banfi, "Estimation of glomerular filtration rate by MDRD equation in athletes: role of body surface area," European Journal of Applied Physiology, vol. 112, no. 1, pp. 201-206, 2012.

[28] A. Grubb, S. Blirup-Jensen, V. Lindström, C. Schmidt, H. Althaus, and I. Zegers, "First certified reference material for Cystatin C in human serum ERM-DA471/IFCC," Clinical Chemistry and Laboratory Medicine, vol. 48, no. 11, pp. 16191621, 2010.

[29] D. Grasso, P. Lanteri, C. Di Bernardo et al., "Salivary steroid hormones response to whole-body cryotherapy in elite rugby players," Journal of Biological Regulators \& Homeostatic Agents. In press.

[30] G. Lombardi, P. Lanteri, S. Porcelli et al., "Hematological profile and martial status in rugby players during whole body cryostimulation," PLoS ONE, vol. 8, no. 2, Article ID e55803, 2013. 

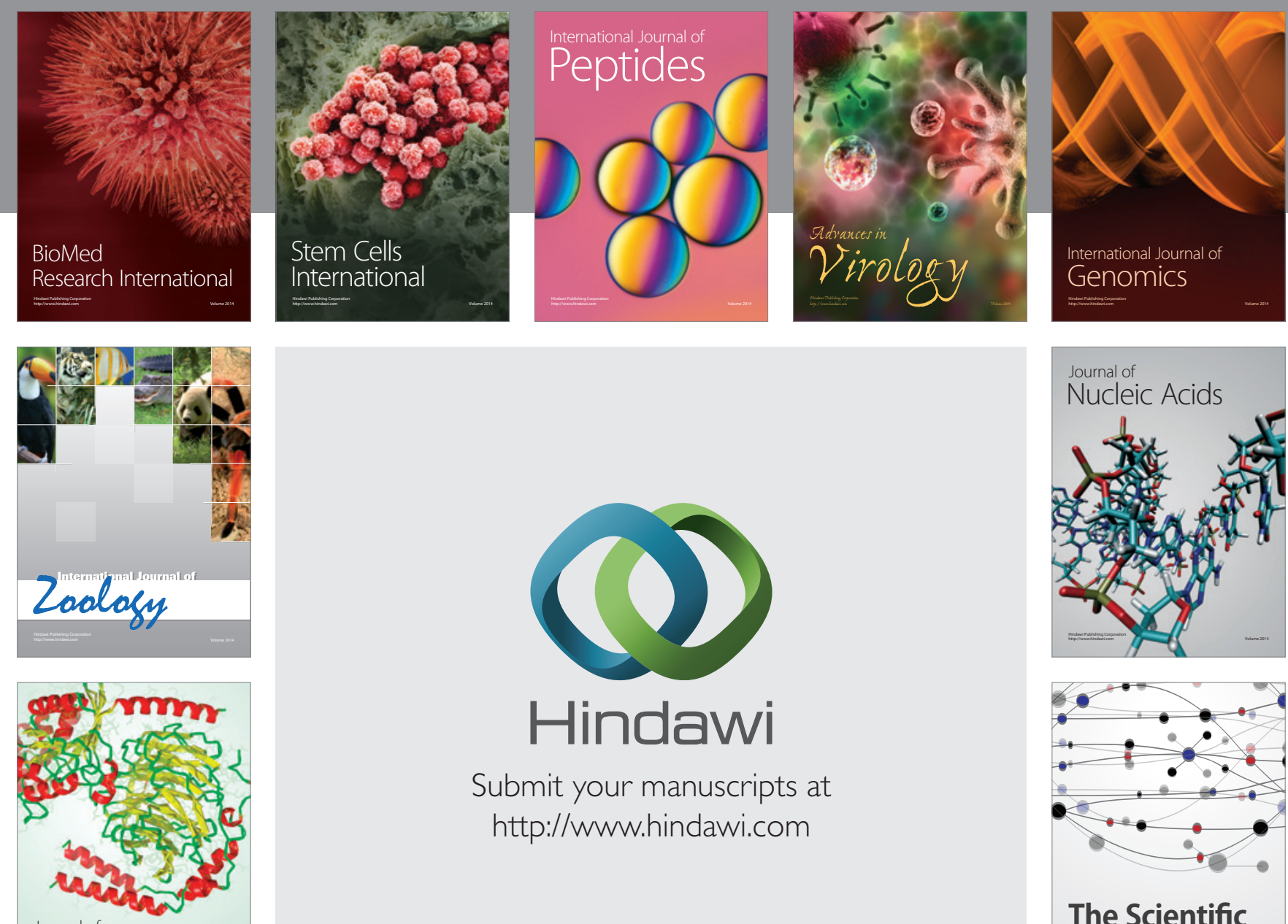

Submit your manuscripts at

http://www.hindawi.com

Journal of
Signal Transduction
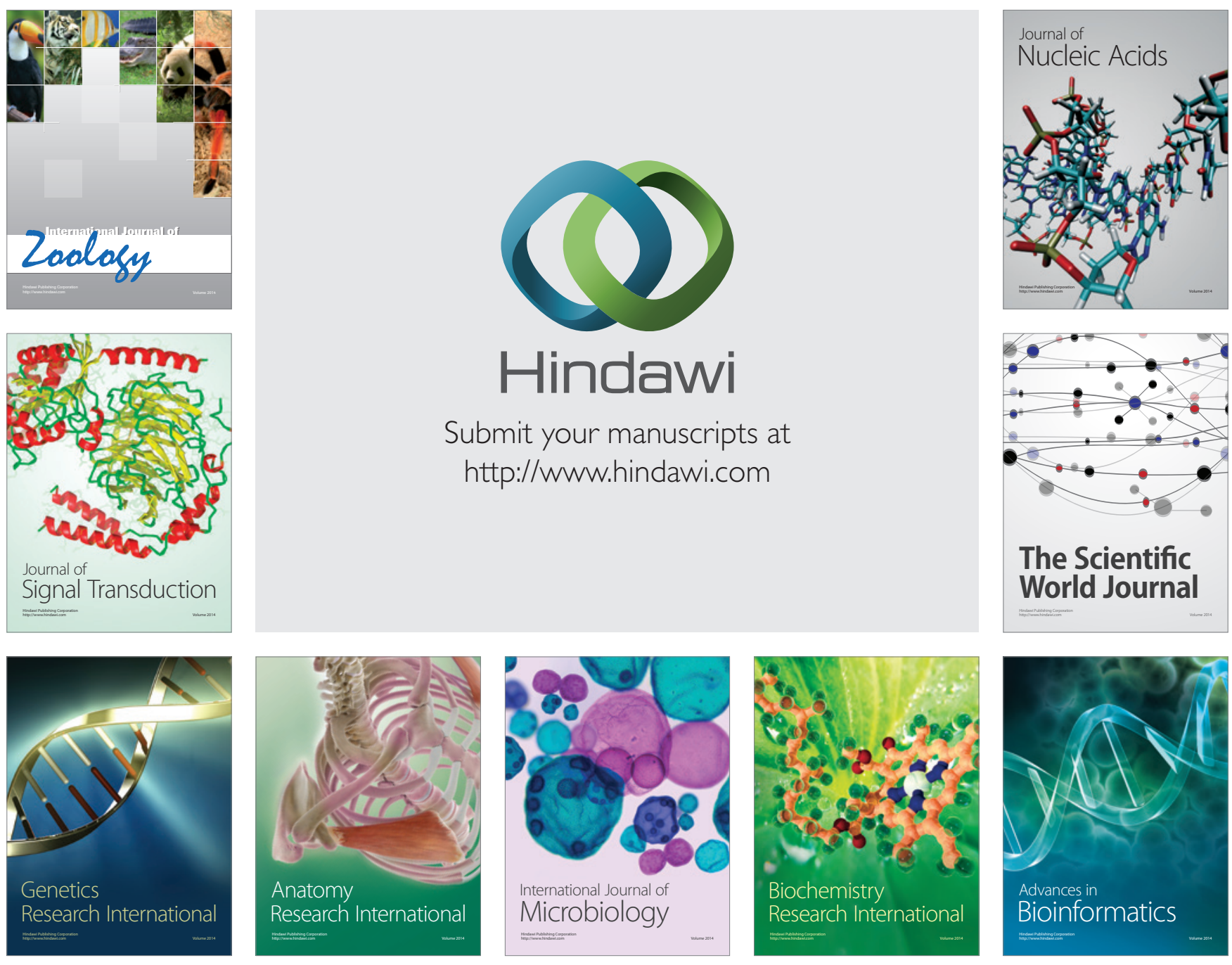

The Scientific World Journal
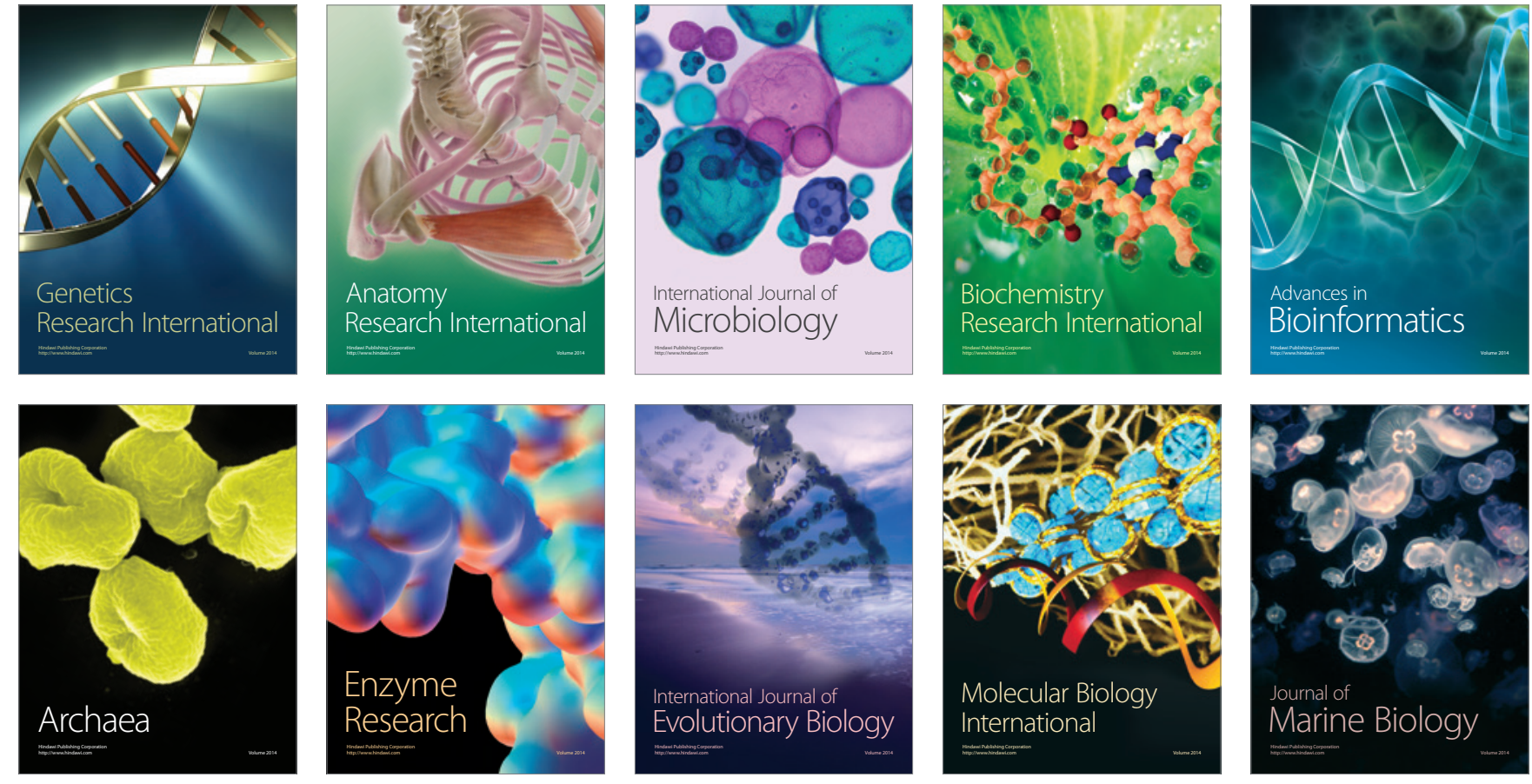